\title{
Resonant Configuration Topology Exploration for Inductive Link Power Transfer
}

\author{
S. Hafiz ${ }^{1}$, Zarimin Zaharudin ${ }^{2}$, Sheroz Khan ${ }^{3}$, Nur Shahida Binti Midi', \\ John Tan Teng Hwang ${ }^{5}$, Kushsairy Abdul Kadir ${ }^{6}$, Jawad Ali Shah ${ }^{7}$ \\ 1,2,3,4 Department Electrical and Computer Engineering, \\ International Islamic University Malaysia, Jalan Gombak, 53100 Kuala Lumpur, Malaysia \\ ${ }^{5,6,7}$ Faculty of Engineering, UCSI University, Kuala Lumpur, Malaysia
}

\begin{tabular}{l}
\hline \hline Article Info \\
\hline Article history: \\
Received Feb 15, 2018 \\
Revised Apr 12, 2018 \\
Accepted Apr 21, 2018
\end{tabular}

\section{Keywords:}

Wireless power

Topology

Power efficiency

Frequency operation

\begin{abstract}
This paper investigates the performance of circuit topology used in wireless power applications to optimize the level of maximum efficiency. We analyse the series and the parallel resonant topologies for use in an inductive coupling link to derive power transfer efficiency expressions verified using MATLAB. We look into the two topologies into the link under resonant conditions for selectively supplying the device with power. The results are obtained analytically which are verified subsequently by MATLAB simulation. We then analyse the links to see how maximum power transfer efficiency for a given pair of coils can be achieved. The topology at a given tuning frequency is used for powering a selected resistive load. The method is presented using a given pair of coils simulated and the results agree well with the theoretical explanation and derivations.
\end{abstract}

Copyright (c) 2018 Institute of Advanced Engineering and Science. All rights reserved.

\section{Corresponding Author:}

S. Hafiz,

Department Electrical and Computer Engineering

International Islamic University Malaysia, Jalan Gombak, 53100 Kuala Lumpur, Malaysia.

Email: sheroz@iium.edu.my

\section{INTRODUCTION}

Inductively coupled coils are used as part of a non-contact link between a source at the manageable point and a load at an inaccessible location [1]. Such inductive links can be used for powering electronic devices, charging up of batteries of UPS, and the battery packs of hybrid electric vehicles [2]. Power can be channelled from a single source to multiple sources or vice versa. Power transferred can be maximized using resonant circuit condition of Series-to-parallel or Series-to-Series or Parallel-to-Parallel circuit configuration. Wireless Power Transfer (WPT) based on the resonant inductive link has attracted a lot of attention in recent times for powering purposes in numerous applications. In bio-medical implants, the partition between the coils and existence of tissues in between the sending and receiving coils are closely coupled. This avoids inserting power cable going through the skin [3]. However, the bio-implants coils in terms of size and separation are way different from those used for charging of electric vehicles. Both applications are prompting research serious interests.

Inductive links need diligent analysis in the light of varying development needs. The main parameter of attention is the power transfer efficiency. By using the magnetic resonant technique in the sending or receiving coils, the efficiency can be improved. It is found that the shunt resonant technique has used in various methods to transfer power efficiency and the shunt method have directed to design and optimize the effort. A large voltage swing is provided by the shunt resonant method to help the rectifier circuitry. However, on the rectifier circuitry, the series resonant method generates more current than voltage and hence places more design constraints. On the other hand, the parallel resonant method has some inherit disadvantages like a poor response to fluctuation coupling and disability to respond better to a smaller load. 
In series, resonant circuit matching network can be used to overcome this drawback. However, this method has some difficulties such as additional cost, space and power loss in the network [4]. Matching network for given application is problematic in vehicles charging due to space issues and because of the gigantic coils size requirements. In cases where additional space is not a big concern, the series resonant method has been used.

Tuning methods are also used for pulse width modulation in inverter applications, impedance network switching, and impedance transformation [5-8]. These methods allow intended circuits to operate conducting in switching condition, zero voltage and derivation of zero voltage in the case of load value. However, these techniques may not be suitable for use in resonant inductive link applications because of the issues related to load and those of the reflected impedance. Since increasing of resistance causes power losses in the coil which are not undesirable. Only the equivalent series resonant coil is added in the inductive link, whereas capacitor is ignored [9]. This paper presents the results of a series circuit inductively coupled resonant circuit serving a resistive load.

\section{TOPOLOGY}

The topology of the Wireless Power Transfer (WPT) is currently becoming essential in view of the ease of understanding the characteristic parameters of wireless technology. In contrast, the power cables limit the portability of numerous electronic gadget devices. There have been research endeavours done for overcoming such limitations. Moreover, many research works have been reported on contact-less battery charging for the movable cellular phone [10-12]. One of the research interests is power transfer capacity from a single source. One point of preferred interest is that in WPT power can be transferred from single transmitter (Tx) to multiple receivers ( $\mathrm{Rx}$ ) or vice versa. With various $\mathrm{Tx}$ and $\mathrm{Rx}$ in a constrained space, the inductive coupling of Tx and Rx with surrounding objects cannot be avoided. For coupled Rx, considering wirelessly power double, LCD on a work area for instance. Since Double screens are normally put one next to the other, non-negligible coupling between them may exist (Figure 1). Comparable circumstances may arise to happen in mobile phone battery charging pads too where numerous phones are wirelessly charged all simultaneously. In manufacturing, plant tools or home appliance vertically stacked secondary coil can be remotely powered by a single Tx on the floor.

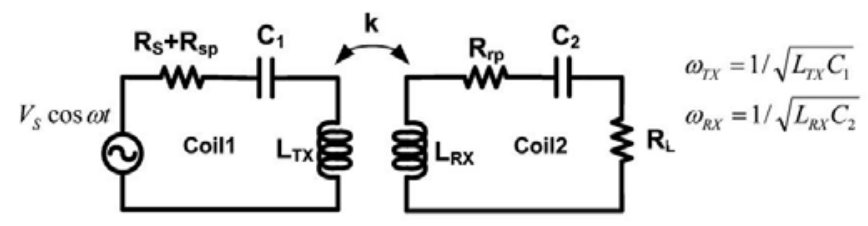

Figure 1. Magnetically coupled resonator $\mathrm{k}$ is the coupling coefficient between the Tx and Rx. Rs and Rl are source and load resistance, respectively, Rsp and Rrp are the parasitic resistances of the Tx and Rx respectively [13]

It could be considerably higher more among primary and secondary coils. Utilizing various Tx is also helpful [14]-[16], the coupling between primary coils likewise cannot be kept away. In wireless power transfer links [17] one case has found (Figure 2). By actuating various hexagonal primaries in a manner that each chose essential isolation with each other, the coupling between primary coils can be minimized.

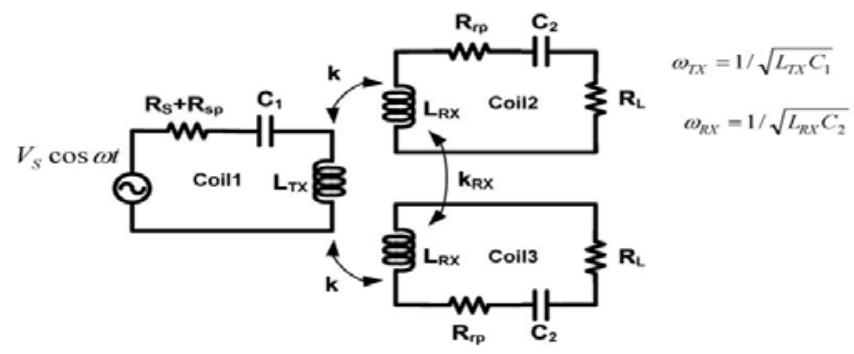

Figure 2. Power transfer forms a single Tx to multiple Rx couple with each other with a coupling coefficient of $\mathrm{k}_{\mathrm{RX}}$ 
The variety of hexagonal primaries empowers free starting and size adjustment of secondary coils. Comparable ideas of Tx cluster for size adjustment can be found in patent [18]. Another advantage of using numerous primary coils is improved power transfer ability under the permitted coupling coefficient, aside from the expected utilization of different primary coil sizes.

However, the outcome of coupling between Tx and between Rx has not been extensively studied. In this paper, we explore the effect of resonant and that of the driving frequencies by maximizing the power transferred between Tx and Rx coils. The performance of numerous Tx on the other hand coupled with single $\mathrm{Rx}$ under the proposed alterations is appeared to be higher than that of a single Tx linked with many Rx coils link. The model conveys $60 \mathrm{~W}$ power at $45-60 \%$ efficiency, even with the extremely lower value of coupling coefficient between Tx and Rx. Beginning from a single Tx/Rx coils pair link, the frequency effects of most extreme efficiency [19] are studied.

\section{PROPOSED METHOD}

The Beginning of single Tx and Rx pair link, the frequency effect of high efficiency is derived. The power transfer efficiency, $\eta$, is determined under tuned operational conditions [20] as in Equation (1):

$$
\begin{aligned}
& \eta=\frac{R_{L}\left|I_{2}\right|^{2}}{\left(R_{s}+R_{s p}\right)\left|I_{1}\right|^{2}+\left(R_{L}+R_{r p}\right)\left|I_{2}\right|^{2}} \\
& =\frac{R_{L}\left|I_{2} / I_{1}\right|^{2}}{\left(R_{s}+R_{s p}\right)+\left(R_{L}+R_{r p}\right)\left|I_{2} / I_{1}\right|^{2}}
\end{aligned}
$$

Where $I_{1}$ and $I_{2}$ are the phasors currents (in rms) of coils 1 and 2. The power delivered to the load is $R_{L}\left|I_{2}\right|^{2}$ while $R_{S}\left|I_{1}\right|^{2}$ is the power supplied by the source, While $R_{s p}$ and $R_{r p}$ are the parasitic resistances of the Tx and Rx loops, respectively. The condition shows that the efficiency is boosted when $\left[I_{2} / I_{1}\right]$ is augmented with the given estimations of $R_{S}, R_{s p}$ and $R_{r p}$. It is intended to find optimized conditions maximizing power exchange efficiency under the given resistance or parasitic resistance values. about $51 \mathrm{~dB}$.

To get, $\left[I_{2} / I_{1}\right], K V L$ is applied to the circuit of Figure 1 . The KVL equivalent equation is (SNR) is

$$
\begin{gathered}
\left(\frac{R_{T X}}{\omega L_{T X}}+1-\frac{\omega^{2} T X}{\omega^{2}}\right) I_{1}+k I_{2} \sqrt{\frac{L_{R X}}{L_{T X}}}=\frac{V_{s}}{\omega L_{T X}} \\
k I_{1} \sqrt{\frac{L_{T X}}{L_{R X}}}+\left(\frac{R_{R X}}{\omega L_{R X}}+1-\frac{\omega^{2}{ }_{R X}}{\omega^{2}}\right) I_{2}=0
\end{gathered}
$$

Where Vs is phasor (in rms) value of the ac source

$$
\mathrm{k}=\mathrm{M} / \sqrt{ } \mathrm{L}_{\mathrm{TX}} \mathrm{L}_{\mathrm{RX}} \text {. }
$$

$\mathrm{M}$ is the mutual inductance

By solving (2) $I_{2} / I_{1}$ is found to be,

$$
\mathrm{R}_{\mathrm{TX}}=\mathrm{R}_{\mathrm{S}}+\mathrm{R}_{\mathrm{SP}} \text { and } \mathrm{R}_{\mathrm{RX}}=\mathrm{R}_{\mathrm{L}}+\mathrm{Rrp} \text {. }
$$

$$
\frac{I_{2}}{I_{1}}=-\frac{k \sqrt{L_{T X} / L_{R X}}}{\frac{R_{R X}}{\omega L_{R X}}+\left(1-\frac{\omega_{R X}{ }^{2}}{\omega^{2}}\right)}
$$

$\mathbf{I}_{\mathbf{2}} / \mathbf{I}_{\mathbf{1}}$ and thus efficiently is, maximized when:

$$
\omega=\frac{\sqrt{2} L_{R X} \omega_{R X}{ }^{2}}{\sqrt{2 L_{R X}^{2} \omega_{R X}^{2}-R_{R X}^{2}}}
$$


The condition stated in (5) is equivalent to amplifying the real part by chopping off the imaginary part of the reflected impedance. This can be seen by examining the aggregate output impedance seen by the ac source:

$$
Z_{\text {OUT }}=R_{T X}+\omega L_{T X}\left(1-\frac{\omega_{T X}^{2}}{\omega^{2}}\right)+\frac{\omega^{2} k^{2} L_{T X} L_{R X} / R_{R X}}{\frac{\omega L_{R X}}{R_{R X}}\left(1-\frac{\omega_{R X}^{2}}{\omega^{2}}\right)+1}
$$

Where the reflected impedance is the term consisting of $\mathrm{k}^{2}$. High efficiency is obtained at $\omega=\omega_{\mathrm{RX}}$, as a result of that the real part of the reflected impedance turns out to be large compared with the parasitic $\mathrm{R}_{\mathrm{TX}}$. On the other hand, when circuits are in parallel resonance we get $\left[V_{2} / V_{1}\right]$ as opposed to $\left[I_{2} / I_{1}\right]$. Take note of that a slight change in the greatest efficiency condition is observed because of the parallel-rapid circuit reflect reactance to the primary side [21-22]. The maximum efficiency condition for the parallel-resonant is found to be as under:

$$
\omega=\frac{\sqrt{2} \omega_{R X} \sqrt{2\left(1-k^{2}\right)-\frac{\left(1-k^{2}\right)^{2}}{Q_{R X}^{2}}}}{2\left(1-k^{2}\right)}
$$

With an approximation of $2 Q_{R X}^{2} \geq\left(1-k^{2}\right)$, the value of $k$ is around or less than 0.1 in a loosely coupled system, and therefore, we mainly consider series-resonant circuit in this paper.

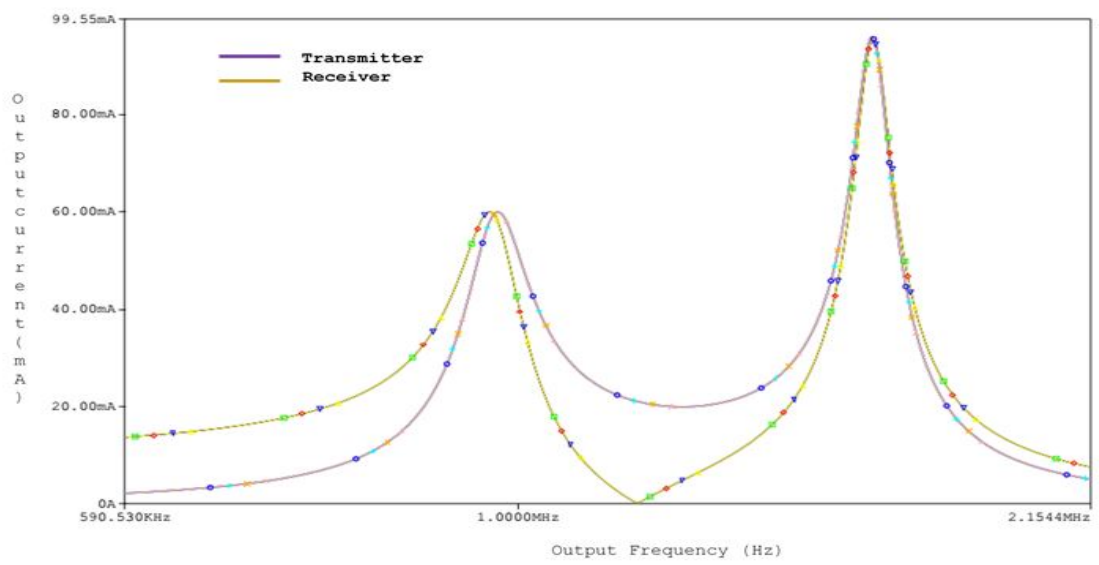

Figure 3. Currents in Tx and Rx coils in over coupled regime. The supply voltage is $20 \mathrm{~V}$, and the coupling coefficient is 0.15 . $\mathrm{L}_{\mathrm{TX}}$ and $\mathrm{L}_{\mathrm{RX}}$ are $39 \mu \mathrm{H}$ and $44 \mu \mathrm{H}$, respectively. $\mathrm{R}_{\mathrm{TX}}$ and $\mathrm{R}_{\mathrm{RX}}$ are 1 and $4.1 \Omega$, respectively. The original resonant frequency of $T_{X}$ and $R_{X}$ is $144 \mathrm{kHz}$ [23]

The conditions adjusted are defined such that $\mathrm{k}>1 / \sqrt{ } \mathrm{QTXQRX}$, where QTX is the Q factor of the Tx, splits the original resonant frequency into two separate frequencies [24-26]. However, the power dissipation in the Tx should also be into account in the efficiency calculation (Figure 3 shows this point). Although the peak Rx power consumption appears at two split frequencies, the Tx power consumption also becomes high at those points. However, at the original Rx resonant frequency of $144 \mathrm{kHz}$, the Tx power dissipation is minimized. Therefore, the frequency for maximum efficiency does not change, even with frequency splitting. However, the power dissemination in the $\mathrm{Tx}$ ought to likewise be considered in the efficiency, figure demonstrates this point. In spite of the fact that the summit Rx control utilization shows up at two split frequencies, the Tx control utilization likewise turns out to be high. In any case, at the Tx control scattering is minimized. Along these lines, the frequency for greatest efficiency does not change. Under the frequency of (4), $\left[\mathrm{I}_{2} / \mathrm{I}_{1}\right]$ becomes: 


$$
\begin{aligned}
& I_{2} / I_{1}=-k \frac{\omega_{R X} L_{R X}}{R_{R X}} \sqrt{\frac{L_{T X}}{L_{R X}}} \\
& =-k Q_{R X} \sqrt{\frac{L_{T X}}{L_{R X}}}
\end{aligned}
$$

\section{RESULTS AND DISCUSSION}

The maximum transmitted power ratio with the distance variation between the Series-Circuits (SC) and Parallel-Circuits (PC) coils, respectively (Figure 4). Figures 5 and 6 show that in Series-to-Series topology the impedance starts from the $\mathrm{k} \Omega$ range, but after a while, the frequency split is clearly visible. Later both the impedance values increase in the same way.

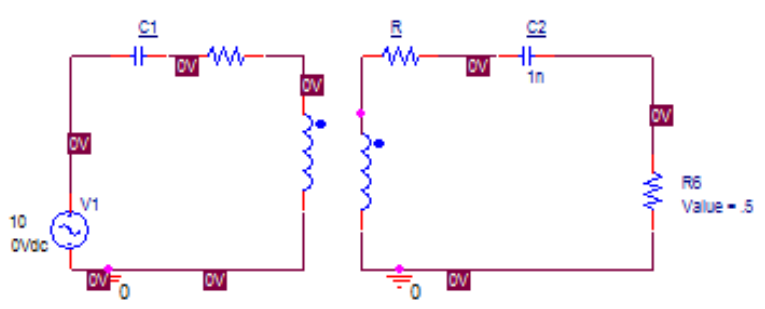

Figure 4. Series-to-series (SS) topology

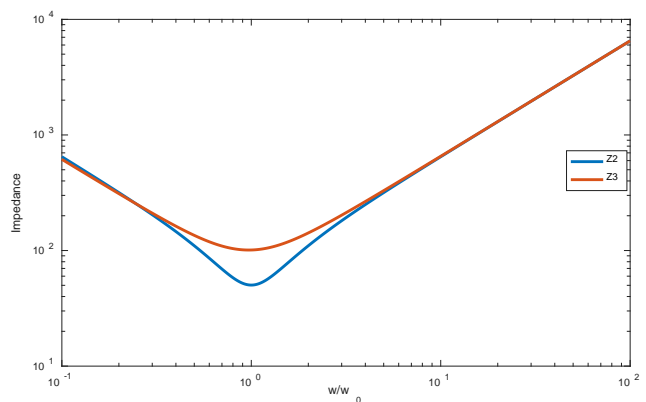

Figure 5. Magnitude plot of Impedance to Series-to-Series topology

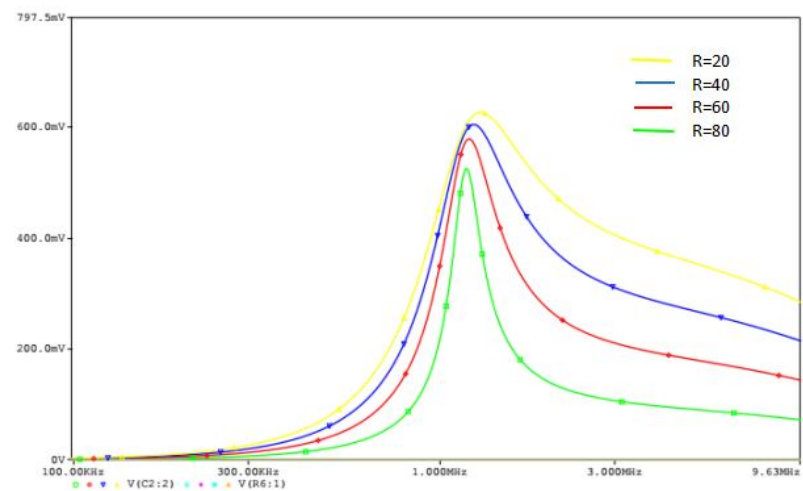

Figure 6. In series to series circuit output voltage with different load values 
Figure 7, 8, 9 and 10 show that in Series-to-Series topology the impedance starts from the $\mathrm{k} \Omega$ range, but after a while, the frequency split is clearly visible. Later both impedance increases in the same way.
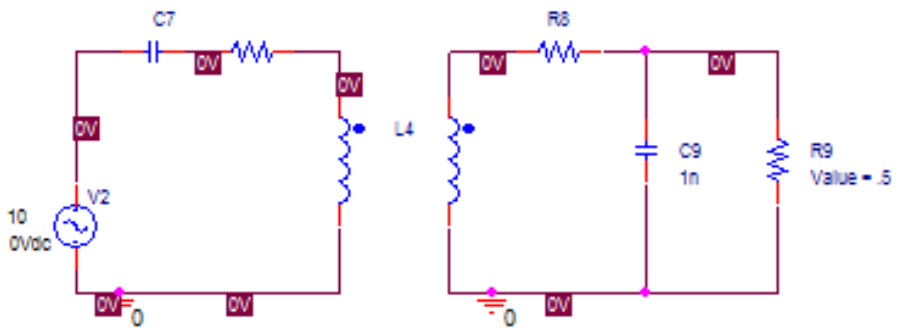

Figure 7. Series to Parallel (SP) topology

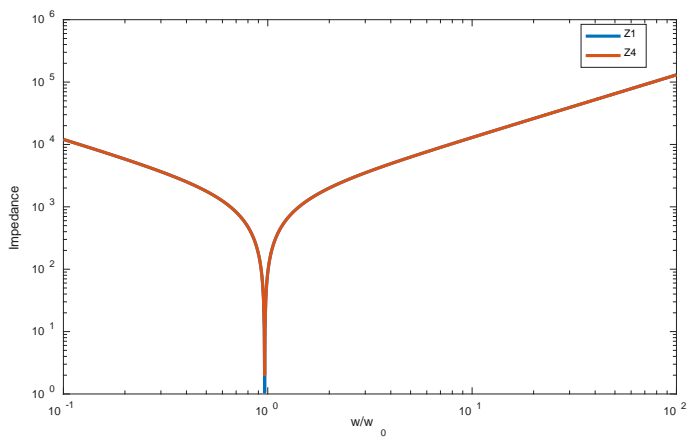

Figure 8. Impedance of series to parallel topology

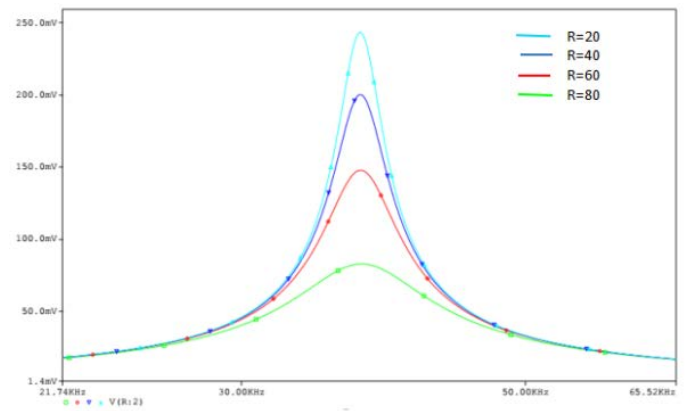

Figure 9. In Series-to-Parallel circuit output voltage with different load values

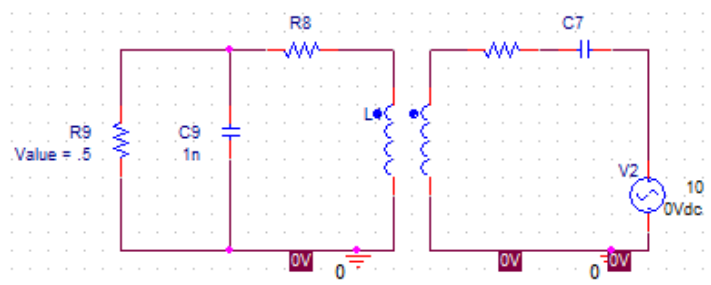

Figure 10. Parallel to Series (PS) topology 


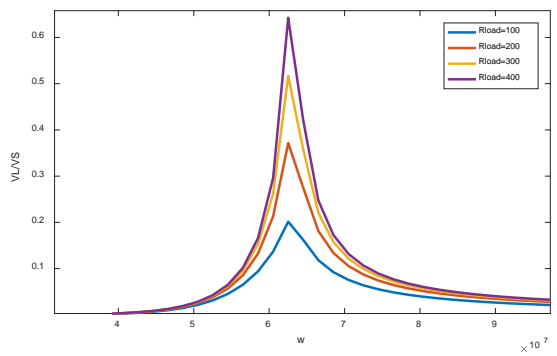

Figure $11 . \mathrm{V}_{\mathrm{L}} / \mathrm{V}_{\mathrm{S}}$ of parallel to series (PS) topology

The Figures 11 and 12 shows a voltage gain of $\left(\mathrm{V}_{\text {load }} / \mathrm{V}_{\text {Source }}\right)$ at critical coupling point at variable parameter.

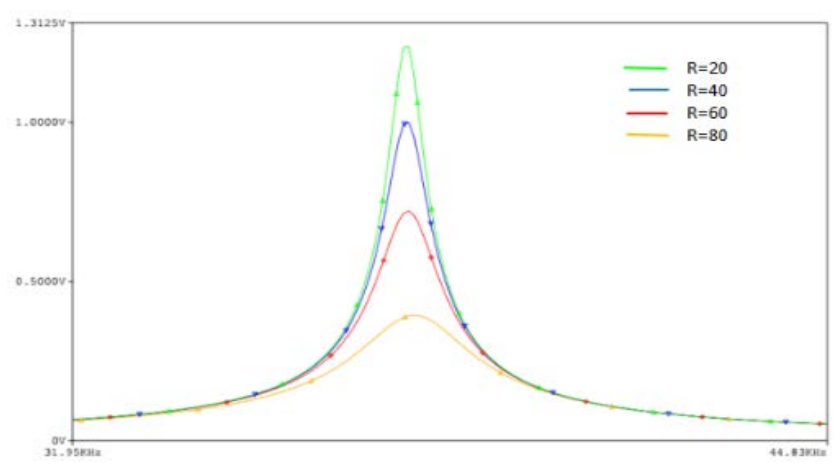

Figure 12. In parallel to series circuit output voltage with different load values.

Again, the investigation has found that the impedance starts from zero and later it hikes sharply to its peaks but later the impedance fall again and continues steadily forward.

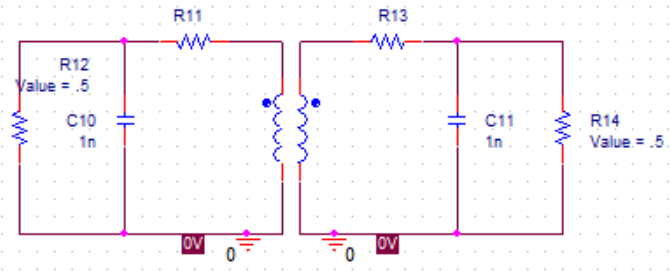

Figure 13. Parallel to Parallel (PP) topology

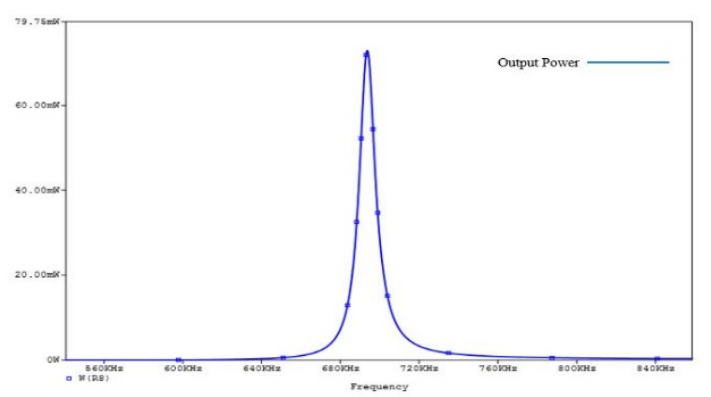


Figure 14. Output power of Parallel to Parallel (PP) Topology

In this analytical work, we can easily find that in parallel-to-parallel topology the impedance is almost zero but later like parallel-to-series the impendence sharply increases and a certain duration impedance it falls sharply (Figure 13, 14 and 15)

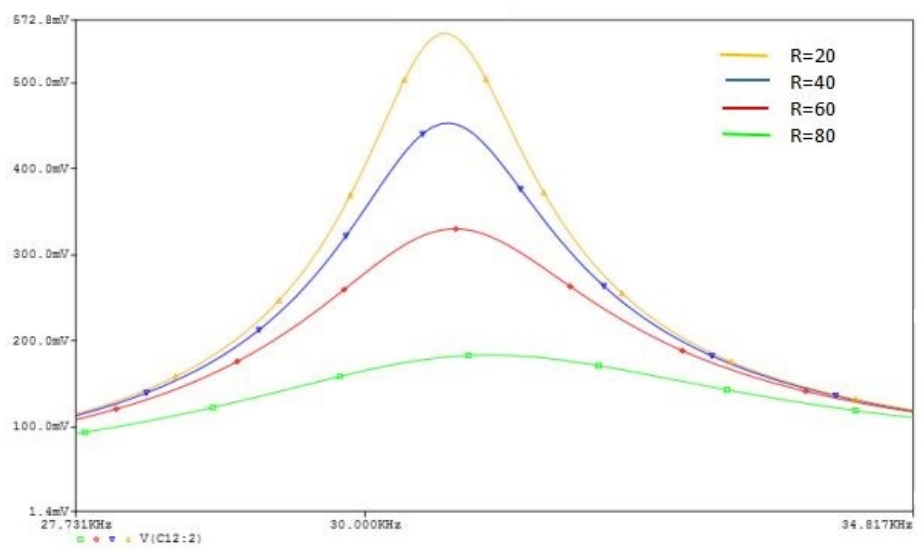

Figure 15. In parallel to parallel circuit output voltage with different load values

The transmitted power proportion as an element of frequency has been at most extreme close to $8 \mathrm{MHz}$, which is the reverberation frequency of both the SC loop and RC coil, with a quality component ranging from 11.3 at a separation of $0.41 \mathrm{~m}$, to 20.7 at a separation of $1.18 \mathrm{~m}$. The most extreme transmitted power proportion dropped quickly as the separation between the SC curl and RC loop is increased.

Figures 4 and 5 demonstrate the transmitted power proportion as a function of frequency, and the greatest transmitted power proportion with the separation variety between the PC loop and SC coil. The separation between the PC coil and LC loop is altered at $1.31 \mathrm{~m}$, and the separation between the RC coil and LC coil was $15 \mathrm{~mm}$. The diameters of the PC coil and LC coil are $0.5 \mathrm{~m}$.

The quality component, which began with an estimation of 20 when the separation between the Primary coil and Secondary coil loop was $0.01 \mathrm{~m}$, demonstrated a most extreme estimation of 200 when the separation between the Primary coil loop and Secondary coil loop was $0.36 \mathrm{~m}$, lastly indicated 114 when the separation between the Primary coil and Secondary coil loop was $0.57 \mathrm{~m}$. The most extreme power transmission proportion demonstrates a comparative pattern with the quality variable achieving a pinnacle estimation of 0.45 when the separation between the Primary coil loop and the Secondary coil has been $0.36 \mathrm{~m}$.

\section{CONCLUSION}

This paper has investigated that the resonant frequencies should be adjusted according to the couplings between Txs or between Rxs. The effectiveness of the proposed adjustments is verified in proof-ofconcept circuits. For maximum efficiency, the driving frequency of Txs should be set to the resonant frequency of the Rxs. To achieve maximum efficiency in this case, the frequency of the Tx should be adjusted to this new effective resonant frequency. The condition for maximum efficiency does not necessarily guarantee maximum power transfer. To maximize the amount of power transfer simultaneously, the effective resonant frequency of Txs should also be set to the effective resonant frequency of Rxs, which is also equal to the driving frequency under the condition of maximum efficiency. The couplings between Txs or between Rxs change these effective resonant frequencies. The couplings between Txs change the effective resonant frequency of Txs, altering the frequency where soft switching is achieved. In this case, the conditions for maximum efficiency and maximum power transfer are simultaneously satisfied. These provide fundamental guidelines for efficient operation in WPT systems with coupled multiple Txs or coupled multiple Rxs.

\section{ACKNOWLEDGEMENt}

Financial assistance for this research by the IIUM Research Management Center (RMC) via RIGS Grant No RIGS 15-147-0147 is highly acknowledged. 


\section{REFERENCES}

[1] Arshad A, Ngah NA, Khan S. Characterization of inductive changes by resonant circuit technique. IEEE conference on Smart Instrumentation, Measurement and Applications (ICSIMA). 2014; 1-4.

[2] Khan, I.M., Khan, S. and Khalifa, O.O. Wireless transfer of power to low power implanted biomedical devices: coil design considerations. IEEE Conference on Instrumentation and Measurement Technology Conference (I2MTC). 2012; $1-5$.

[3] Habib, M.S., Rahman, M.M., Arshad, A. and Khan, S. Analysis of Power Transfer Efficiency of Inductive Coupled Telemetry System for Wireless Power Transfer. IEEE Conference on Computer and Communication Engineering (ICCCE). 2014; 32-35.

[4] Jegadeesan, R. and Guo, Y.X. Topology selection and efficiency improvement of inductive power links. IEEE Transactions on Antennas and Propagation. 2012; 60(10); 4846-4854.

[5] Huh, J., Lee, S.W., Lee, W.Y., Cho, G.H. and Rim, C.T. Narrow-width inductive power transfer system for online electrical vehicles. IEEE Transactions on Power Electronics. 2011; 26(12); 3666-3679.

[6] Budhia, M., Covic, G.A. and Boys, J.T. Design and optimization of circular magnetic structures for lumped inductive power transfer systems. IEEE Transactions on Power Electronics. 2011; 26(11); 3096-3108.

[7] Wu, H.H., Covic, G.A., Boys, J.T. and Robertson, D.J. A series-tuned inductive-power-transfer pickup with a controllable AC-voltage output. IEEE Transactions on Power Electronics. 2011; 26(1); 98-109.

[8] Van Schuylenbergh, K. and Puers, R. Inductive powering: basic theory and application to biomedical systems. Springer Science \& Business Media. 2009.

[9] Aldhaher, S., Luk, P.C.K. and Whidborne, J.F. Tuning class E inverters applied in inductive links using saturable reactors. IEEE Transactions on Power Electronics. 2014; 29(6); 2969-2978.

[10] Jang, Y. and Jovanovic, M.M. A contactless electrical energy transmission system for portable-telephone battery chargers. IEEE Transactions on Industrial Electronics. 2003; 50(3), 520-527.

[11] Choi, B., Nho, J., Cha, H., Ahn, T. and Choi, S. Design and implementation of low-profile contactless battery charger using planar printed circuit board windings as energy transfer device. IEEE Transactions on Industrial Electronics. 2004; 51(1); 140-147.

[12] Hirai, J., Kim, T.W. and Kawamura, A. Study on intelligent battery charging using inductive transmission of power and information. IEEE Transactions on Power Electronics. 2000; 15(2); 335-345.

[13] Li, H.L., Hu, A.P., Covic, G.A. and Tang, C.S. Optimal coupling condition of IPT system for achieving maximum power transfer. Electronics Letters. 2009; 45(1); 76-77.

[14] Hatanaka, K., Sato, F., Matsuki, H., Kikuchi, S., Murakami, J.I., Kawase, M. and Satoh, T. Power transmission of a desk with a cord-free power supply. IEEE Transactions on Magnetics. 2002; 38(5); 3329-3331.

[15] Wireless Power Consortium. System description wireless power transfer. Volume I: Low Power, Part, 1. 2010.

[16] Ahn, D. and Hong, S. Effect of coupling between multiple transmitters or multiple receivers on wireless power transfer. IEEE Transactions on Industrial Electronics. 2013; 60(7); 2602-2613.

[17] Wireless Power Consortium. System description wireless power transfer. Volume I: Low Power, Part, 1. 2010.

[18] Giler, E.R., Hall, K.L., Kesler, M.P., Soljacic, M., Karalis, A., Kurs, A.B., Li, Q. and Ganem, S.J., Witricity Corporation, 2013. Wireless energy transfer using repeater resonators. U.S. Patent 8,587,155.

[19] Ahn, D. and Hong, S. Effect of coupling between multiple transmitters or multiple receivers on wireless power transfer. IEEE Transactions on Industrial Electronics. 2013; 60(7); 2602-2613.

[20] Kurs, A., Karalis, A., Moffatt, R., Joannopoulos, J.D., Fisher, P. and Soljačić, M. Wireless power transfer via strongly coupled magnetic resonances. Science. 2007; 317(5834); 83-86.

[21] Wang, C.S., Stielau, O.H. and Covic, G.A. Design considerations for a contactless electric vehicle battery charger. IEEE Transactions on industrial electronics. 2005; 52(5); 308-1314.

[22] Lee, S., Huh, J., Park, C., Choi, N.S., Cho, G.H. and Rim, C.T. On-line electric vehicle using inductive power transfer system. In Energy Conversion Congress and Exposition (ECCE). 2010; 1598-1601.

[23] Ahn, D. and Hong, S. Effect of coupling between multiple transmitters or multiple receivers on wireless power transfer. IEEE Transactions on Industrial Electronics. 2013; 60(7); 2602-2613.

[24] Sample, A.P., Meyer, D.T. and Smith, J.R. Analysis, experimental results, and range adaptation of magnetically coupled resonators for wireless power transfer. IEEE Transactions on Industrial Electronics. 2011; 58(2); 544-554.

[25] Baek, D., Song, T., Ko, S., Yoon, E. and Hong, S. Analysis on resonator coupling and its application to CMOS quadrature VCO at 8 GHz. In Radio Frequency Integrated Circuits (RFIC) Symposium. 2003; 85-88.

[26] Imura, T. and Hori, Y. Maximizing air gap and efficiency of magnetic resonant coupling for wireless power transfer using equivalent circuit and Neumann formula. IEEE Transactions on industrial electronics. 2011; 58(10); 47464752 .

[27] Ahmed Younes Shdefat, Moon-Il Joo, Sung-Hoon Choi, Hee-Cheol Kim, "Utilizing ECG Waveform Features as New Biometric Authentication Method," International Journal of Electric \& Computer Engineering, Vol. 8, No. 2, April 2018

[28] D Istardi, A Triwinarko. Induction Heating Process Design Using COMSOL ${ }^{\circledR}$ Multiphysics Software. TELKOMNIKA (Telecommunication, Computing, Electronics and Control). 2011; 9(2): 327-334. 